\title{
Efficacy, safety and tolerability of GSK2190915, a 5-lipoxygenase activating protein inhibitor, in adults and adolescents with persistent asthma: a randomised dose-ranging study
}

Richard MA Follows ${ }^{1 *}$, Neil G Snowise ${ }^{1,4}$, Shu-Yen Ho ${ }^{2}$, Claire L Ambery ${ }^{1}$, Kevin Smart ${ }^{3,5}$ and Barbara A McQuade ${ }^{1}$

\begin{abstract}
Background: GSK2190915 is a high affinity 5-lipoxygenase-activating protein inhibitor being developed for the treatment of asthma. The objective of this study was to evaluate GSK2190915 efficacy, dose-response and safety in subjects with persistent asthma treated with short-acting beta2-agonists (SABAs) only.

Methods: Eight-week multicentre, randomised, double-blind, double-dummy, stratified (by age and smoking status), parallel-group, placebo-controlled study in subjects aged $\geq 12$ years with a forced expiratory volume in 1 second $\left(\mathrm{FEV}_{1}\right)$ of $50-85 \%$ predicted. Subjects $(n=700)$ were randomised to receive once-daily $(\mathrm{QD})$ oral GSK2190915 (10-300 mg), twice-daily inhaled fluticasone propionate $100 \mu \mathrm{g}$, oral montelukast $10 \mathrm{mg}$ QD or placebo. The primary endpoint was mean change from baseline (randomisation) in trough (morning pre-dose and pre-rescue bronchodilator) $\mathrm{FEV}_{1}$ at the end of the 8-week treatment period. Secondary endpoints included morning and evening peak expiratory flow, symptom-free days and nights, rescue-free days and nights, day and night-time symptom scores, day and night-time rescue medication use, withdrawals due to lack of efficacy, Asthma Control Questionnaire and Asthma Quality of Life Questionnaire scores.
\end{abstract}

Results: For the primary endpoint, there was no statistically significant difference between any dose of GSK2190915 QD and placebo. However, repeated measures sensitivity analysis demonstrated nominal statistical significance for GSK2190915 $30 \mathrm{mg}$ QD compared with placebo (mean difference: 0.115 L [95\% confidence interval: 0.00, 0.23], $p=0.044$ ); no nominally statistically significant differences were observed with any of the other doses. For the secondary endpoints, decreases were observed in day-time symptom scores and day-time SABA use for GSK2190915 $30 \mathrm{mg}$ QD versus placebo ( $\mathrm{s}$ 0.05). No dose-response relationship was observed for the primary and secondary endpoints across the GSK2190915 dose range studied; the $10 \mathrm{mg}$ dose appeared to be sub-optimal. GSK2190915 was associated with a dose-dependent reduction in urinary leukotriene $\mathrm{E}_{4}$. The profile and incidence of adverse events were similar between treatment groups.

Conclusion: Efficacy was demonstrated for GSK2190915 30 mg compared with placebo in day-time symptom scores and day-time SABA use. No additional improvement on efficacy endpoints was gained by administration of GSK2190915 doses greater than 30 mg. GSK2190915 was well-tolerated. These results may support further studies with GSK2190915 $30 \mathrm{mg}$.

Trial registration: Clinicaltrials.gov: NCT01147744.

Keywords: GSK2190915, FLAP, Asthma, Efficacy, Safety, Tolerability, Dose-ranging

\footnotetext{
* Correspondence: richard.m.follows@gsk.com

'GlaxoSmithKline, Stockley Park West, Uxbridge, Middlesex UB11 1BT, UK

Full list of author information is available at the end of the article
} 


\section{Background}

Leukotrienes (LTs) are produced by mast cells, eosinophils, macrophages and neutrophils in response to allergic or inflammatory stimuli [1-3]. They are products of the 5-lipoxygenase (5-LO) pathway of arachidonic acid metabolism and their synthesis is initiated by $5-\mathrm{LO}$ in concert with 5-lipoxygenase-activating protein (FLAP). This part of the pathway divides into two branches: one leading to production of $\mathrm{LTB}_{4}$ and the other to the production of the cysteinyl LTs (cysLTs) $\mathrm{LTC}_{4}, \mathrm{LTD}_{4}$ and $\mathrm{LTE}_{4}$, so-called because they contain the amino acid cysteine conjugated to their lipid backbone. The central role of LTs in asthma pathogenesis is well-established as they function as potent mediators of inflammation and airway constriction [4-7]. Concentrations of the products of the 5-LO pathway are increased in patients with asthma of all severities despite treatment with corticosteroids [8-10], the cornerstone anti-inflammatory therapy in asthma.

Leukotriene $\mathrm{B}_{4}$ is a potent chemoattractant of neutrophils [11]. Irrespective of therapy with corticosteroids, $\mathrm{LTB}_{4}$ concentrations were increased in the bronchoalveolar fluid, sputum and tissue of patients with severe asthma compared with non-asthmatic control subjects and patients with mild-moderate asthma [10,12]. Neutrophil numbers have been shown to increase in patients with severe asthma compared with milder asthma phenotypes, and neutrophilic inflammation is resistant to the effects of corticosteroids $[9,12,13]$. The cysLTs are potent constrictors of airway smooth muscle, they increase vascular permeability and serve as chemoattractants for eosinophils [4-6]. Cysteinyl LTs are directly involved in bronchoconstriction, airway oedema and mucus secretion, characteristic of the asthmatic phenotype [14]. Inhibition of cysLTs by the administration of the cysLT receptor antagonist montelukast elicits bronchodilatory effects and significantly reduces eosinophil numbers in the peripheral blood and induced sputum of patients with asthma [15]. In addition, administration of the 5-LO inhibitor zileuton to patients with severe asthma led to improvements in lung function [16]. These observations indicate that inhibition of the production of LTs via the two branches of the 5-LO pathway could confer beneficial effects on patients with persistent asthma.

GSK2190915 is a high affinity FLAP inhibitor that attenuates the production of LTs through inhibition of the first step of the 5-LO pathway. In vitro and in vivo studies demonstrated that GSK2190915 reproducibly inhibited the production of both $\mathrm{LTB}_{4}$ and cysLTs [17]. In healthy subjects, GSK2190915 was well-tolerated with a systemic exposure that increased in a dose-related manner [18]. GSK2190915 also demonstrated dosedependent inhibition of blood $\mathrm{LTB}_{4}$ production and of urinary excretion of the cysLT, $\mathrm{LTE}_{4}$ [18]. The primary objective of the current study was to evaluate the efficacy, dose-response, safety and tolerability of GSK2190915 administered once-daily (QD) over an 8-week period in adolescents and adults with persistent uncontrolled asthma receiving a short-acting beta2-agonist (SABA). The secondary objective of this study was to explore the efficacy of GSK2190915 against established asthma treatments, namely montelukast and the inhaled corticosteroid (ICS) fluticasone propionate (FP).

\section{Methods \\ Subjects}

Subjects aged 12 years or older were eligible for enrolment if they had a diagnosis of asthma (as defined by the National Institutes of Health [19]) with a best (the highest of three technically acceptable measurements) pre-bronchodilator forced expiratory volume in 1 second $\left(\mathrm{FEV}_{1}\right)$ of $50-85 \%$ of the predicted normal value, and reversibility of at least $12 \%$ and $200 \mathrm{~mL}$ within 30 minutes after inhaled salbutamol/albuterol. The original protocol also allowed males to be recruited to the study. However, the protocol was amended to include only females after findings of testicular toxicity in rats at high exposures of GSK2190915 following 6-month dosing were reported during the conduct of the study. Former and current smokers, with a smoking history of $\leq 10$ pack years, were required to demonstrate a post-salbutamol/albuterol $\mathrm{FEV}_{1}$ /forced vital capacity ratio of $>0.70$ to exclude subjects with fixed airways. Eligible subjects were required to have been taking a SABA for at least 3 months before screening. They were also required to be able to replace their SABA with salbutamol/albuterol to be used as rescue medication during the run-in and treatment periods. Other permitted medications included stabledose immunotherapy, intranasal corticosteroids and short and long-acting antihistamines.

Exclusion criteria at screening included a history of life-threatening asthma (defined as an asthmatic episode that had required intubation and/or was associated with hypercapnoea, respiratory arrest or hypoxic seizures within the last 5 years), an asthma exacerbation requiring oral corticosteroids in the 3 months prior to screening or hospitalisation for asthma in the previous 6 months, an unresolved infection in the past 4 weeks leading to a change in asthma management or that affected the subject's asthma status or ability to participate in the study, use of ICSs in the past 6 weeks or of systemic, oral or depot corticosteroids in the past 12 weeks. Non-smoking subjects were not permitted to have used tobacco products within 6 months of screening. Subjects were also excluded if they had received statins or other organic anion transport protein $1 \mathrm{~B} 1$ substrates within 4 weeks of screening or had any adverse reaction including immediate or delayed 
hypersensitivity to any beta2-agonist, sympathomimetic drug, intranasal, inhaled or systemic corticosteroid.

\section{Study design}

This was a Phase IIb, multicentre, randomised, doubleblind, double-dummy, parallel-group, placebo-controlled study that was conducted from January 2010 to October 2011 at 89 sites in six countries (GlaxoSmithKline protocol: LPA112186; Clinicaltrials.gov registration number: NCT01147744). All subjects provided signed informed consent prior to screening. Local Ethics Review Committees provided approval for the study, which was conducted in accordance with Good Clinical Practice, applicable country-specific requirements and the guiding principles of the Declaration of Helsinki (2008).

Eligible subjects completed a 2 -week pre-treatment run-in period during which they were required to attend the clinical unit for assessment of their adherence with an electronic daily diary (eDiary, AM3 device by ERT, Bavaria, Germany). At the end of the study run-in period, subjects were eligible to enter the treatment period if they had a morning pre-dose $\mathrm{FEV}_{1}$ between $50 \%$ and $85 \%$ of their predicted normal and had either any combination of the daily asthma symptom scores (day-time plus night-time) of $\geq 1$, or used salbutamol/albuterol on at least 4 days of the last 7 consecutive days of the run-in period.

Subjects were randomised to receive GSK2190915 tablets (10 mg, $30 \mathrm{mg}, 100 \mathrm{mg}, 300 \mathrm{mg}$ ) QD (morning), FP $100 \mu \mathrm{g}$ twice-daily (BID; morning and evening) via a Diskus $^{\text {Tा }} /$ Accuhaler $^{\text {TM }}$ (GlaxoSmithKline, Ware, UK) and montelukast $10 \mathrm{mg}$ capsules QD (evening). A double dummy design was used to ensure blinding to treatment (see Additional file 1). Subjects received treatment on an out-patient basis for 8 weeks. Subjects aged 12-14 years were not randomised to the montelukast arm as the $10 \mathrm{mg}$ dose is not licensed for this age group. Randomisation was stratified according to subject age and smoking status. Follow-up took place 1 week after completion of the treatment period. Following their withdrawal, all male subjects were offered a clinical assessment.

Subjects were withdrawn from study medication due to 'lack of efficacy' if they changed their asthma medication, had an asthma exacerbation (defined as worsening asthma requiring any treatment other than rescue salbutamol/albuterol) or had signs of asthma instability (defined as a fall in clinic $\mathrm{FEV}_{1}$ to $<80 \%$ of value at randomisation, peak expiratory flow $[\mathrm{PEF}]<80 \%$ of the mean run-in value on more than 3 days between consecutive visits or use of salbutamol/albuterol $\geq 12$ inhalations/day on more than 2 days between consecutive visits).

Adherence to treatment was assessed from Week 4 until Week 8 (or early withdrawal) by counting returned tablets, capsules and reviewing the dosing counter on the Diskus/Accuhaler. Allocation to treatment group was determined according to a computer generated schedule; numbered containers were used to implement allocation. Neither the subject nor the investigator knew which study medication the subject was receiving.

\section{Efficacy assessments}

Assessments of efficacy were based on measures of spirometry, including trough $\mathrm{FEV}_{1}$ (pre-bronchodilator and pre-dose); parameters recorded in the daily diary including symptoms, use of rescue medication and daily PEF; questionnaires including Asthma Control Questionnaire (ACQ)-6 and the Asthma Quality of Life Questionnaire (AQLQ) (+12).

The primary endpoint of the study was mean change from baseline (randomisation) in trough (morning predose and pre-rescue bronchodilator) $\mathrm{FEV}_{1}$ at the end of the 8-week treatment period. Changes in $\mathrm{FEV}_{1}$ at weeks 1, 2, 4 and 6 were also assessed. Efficacy measures for secondary endpoints recorded by the patients using the daily diary were: mean change from baseline in daily trough (pre-dose and pre-rescue bronchodilator) morning and evening PEF averaged over the 8-week treatment period; mean change from baseline in the percentage of symptom-free days and nights and rescuefree days and nights; mean change from baseline in day and night-time symptom scores; mean change from baseline in day and night-time rescue salbutamol/albuterol use; withdrawals due to lack of efficacy and mean change from baseline in ACQ and AQLQ scores. In addition, other endpoints were assessed: symptom-free 24-hour periods, rescue-free 24-hour periods, 24-hour period symptom scores and 24-hour SABA use.

All post-randomisation $\mathrm{FEV}_{1}$ measurements were taken within 1 hour of the time $\mathrm{FEV}_{1}$ was measured at randomisation. Before attending the clinical unit for lung function assessments, subjects had to withhold their rescue medication for at least 6 hours. At weeks 1, 2, 4, 6 and $8, \mathrm{FEV}_{1}$ was measured approximately 24 hours postmorning dose and approximately 12 hours post-evening dose.

\section{Pharmacokinetic/pharmacodynamic assessments}

Blood samples for pharmacokinetic analysis were collected at pre-dose on weeks 0,2 and 4. Post-dose samples were collected on weeks 1 and 8 within the following time windows: first sample: $0.5-2$ hours; second sample: 2-5 hours. Plasma samples from subjects who received only GSK2190915 were analysed using a validated analytical method based on protein precipitation followed by high performance liquid chromatography with tandem mass spectrometry analysis. The lower limit of 
quantification for GSK2190915 was $5 \mathrm{ng} / \mathrm{mL}$ and the higher limit of quantification was $5000 \mathrm{ng} / \mathrm{mL}$.

Three spot urine samples were collected at Week 0 and pre-dose on weeks 1 and 8 to determine mean change from baseline in urinary $\mathrm{LTE}_{4}$. Urine samples were analysed for $\mathrm{LTE}_{4}$ using a validated analytical method based on solid-phase extraction followed by high performance liquid chromatography with tandem mass spectrometric detection. The lower limit of quantification for $\mathrm{LTE}_{4}$ was $5 \mathrm{pg} / \mathrm{mL}$ and the higher limit of quantification was $1000 \mathrm{pg} / \mathrm{mL}$.

\section{Safety and tolerability measurements}

Safety and tolerability were assessed by monitoring adverse events during the treatment period until follow-up, and serious adverse events from screening to follow-up. Adverse events were coded using the Medical Dictionary for Regulatory Activities (MedDRA). Asthma exacerbations were recorded. Assessments of liver function, standard laboratory parameters, vital signs (pulse rate and systolic and diastolic blood pressure) and 12-lead electrocardiograms (ECGs) were also performed.

\section{Statistical analysis}

The primary hypothesis of the study was that $\mathrm{FEV}_{1}$ in the GSK2190915 groups would demonstrate a significant increase at the end of the 8-week treatment period compared with that in the placebo group. Assuming a between-subject common standard deviation in $\mathrm{FEV}_{1}$ of $415 \mathrm{~mL}$, it was estimated that it would be necessary to recruit 630 subjects (90 per group) to provide $89 \%$ power to detect a difference (two-sided 5\% level) of $200 \mathrm{~mL}$ in pairwise comparisons between any GSK2190915 dose group and placebo.

A sequential testing procedure was followed to account for multiplicity from multiple pairwise comparisons: statistical comparison of the highest GSK2190915 dose with placebo was performed first and subsequent comparisons at lower doses continued in a sequential manner. A 5\% level of statistical significance was claimed only if the preceding comparison was significant at the $5 \%$ level. Any other test results were not adjusted for multiplicity and any p-value $\leq 0.05$ from those testing results was interpreted as nominally significant. Primary efficacy analysis was performed using analysis of covariance (ANCOVA) with a last observation carried forward approach used to impute missing data; repeat measures model was also performed for sensitivity analysis.

Secondary efficacy analysis was performed using ANCOVA, except for the comparison between each treatment group and placebo for the number of withdrawals due to lack of efficacy in which a Fisher's Exact test was used. No multiplicity adjustments were made on the secondary efficacy endpoints. Any $p$ value $\leq 0.05$ was identified as nominally significant. The comparisons between active treatments were only for exploratory purposes. Population pharmacokinetic analysis of sparse GSK2190915 plasma concentration-time data was performed using non-linear mixed effects modelling as was the population pharmacokinetic-pharmacodynamic analysis to assess the plasma GSK2190915 concentration versus urinary $\mathrm{LTE}_{4}$ relationship. Non-quantifiable pharmacokinetic and pharmacodynamic data were treated as missing for the population analysis. No formal statistical analysis was performed on safety data. For all endpoints, data are reported for subjects who received at least one dose of study medication.

\section{Results \\ Subjects}

Of 1245 subjects screened, 700 were randomised and 548 completed the study (Figure 1). One hundred and thirteen (16\%) subjects, including 56 males, were randomised under the original protocol. However, after the protocol was amended to withdraw males and include females only, an additional 587 (84\%) female subjects were subsequently recruited. One hundred and fifty-two (22\%) subjects withdrew from the study and the most frequent reasons for withdrawal were: lack of efficacy (70 subjects), decision to withdraw all male subjects (33 subjects) and withdrawal of consent (25 subjects). The numbers of subjects in each group withdrawing from the study were similar. Demographic and baseline subject characteristics were well-matched across treatment groups (Table 1).

\section{Efficacy}

At the end of the 8-week treatment period, there was no statistically significant difference between any dose of GSK2190915 QD and placebo for mean change from baseline in trough (morning pre-dose and pre-rescue bronchodilator) $\mathrm{FEV}_{1}$ (Figure 2). Similar results were obtained for montelukast $10 \mathrm{mg}$ QD compared with placebo. However, mean difference in change from baseline between FP $100 \mu \mathrm{g}$ BID and placebo achieved nominal statistical significance (Figure 2). The repeated measures sensitivity analysis of change from baseline in trough $\mathrm{FEV}_{1}$ indicated an overall increase for all doses of GSK2190915 (Figure 3) with the greatest increase observed for FP $100 \mu \mathrm{g}$ BID. With the repeated measures analysis, treatment differences (active minus placebo) achieved nominal statistical significance for GSK2190915 $30 \mathrm{mg}$ QD at Week 8 (least squares [LS] mean difference: 0.115 L [95\% confidence interval, CI: 0.00, 0.23], $\mathrm{p}=0.044)$. For FP $100 \mu \mathrm{g}$ BID, nominal statistical significance (active minus placebo) was achieved at all timepoints (Weeks 1 to 8 ). 


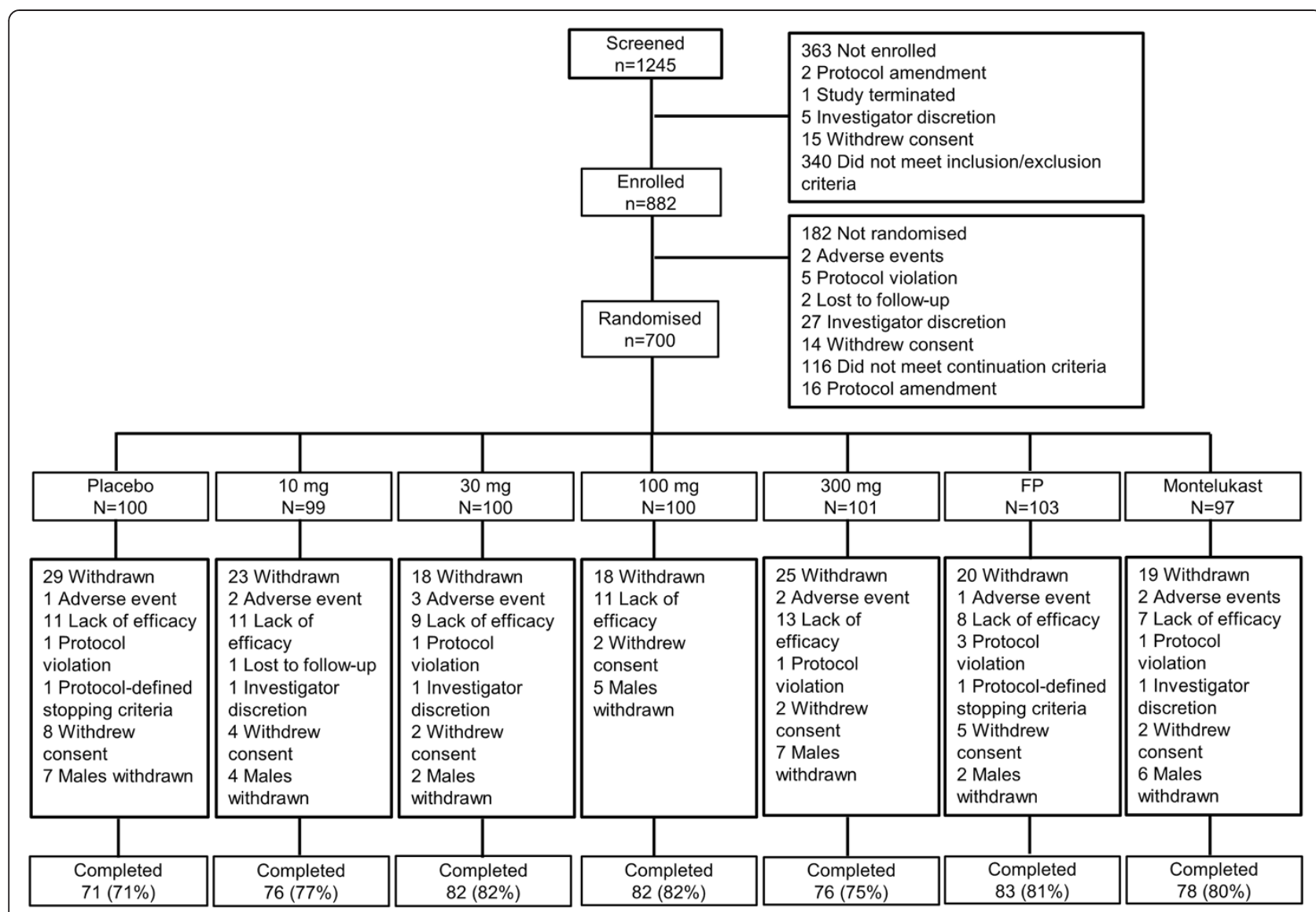

Figure 1 CONSORT diagram. Subject flow through study. FP = fluticasone propionate.

There were no statistically significant differences for any dose of GSK2190915 versus placebo in the secondary endpoints of morning and evening PEF, symptom-free days and nights, rescue-free days and nights, night-time symptom scores and night-time SABA use (Additional file 2); similar results were observed for montelukast $10 \mathrm{mg}$ QD versus placebo. However, there were nominally statistically significant differences for FP $100 \mu \mathrm{g}$ versus placebo for symptom-free days and rescue-free days. Nominally statistically significant reductions were observed for comparisons between GSK2190915 $30 \mathrm{mg}$ QD and placebo for the secondary endpoints of day-time symptom scores and day-time SABA use (Additional file 2). Comparisons between montelukast $10 \mathrm{mg}$ QD or FP $100 \mu \mathrm{g}$ and placebo showed a nominally statistically significant decrease for day-time SABA use (Additional file 2). Total scores for ACQ and total and domain scores for AQLQ were similar for placebo, GSK2190915 (any dose) and montelukast $10 \mathrm{mg}$ QD whilst a nominally statistically significant decrease was observed for FP $100 \mu \mathrm{g}$ versus placebo (Additional file 2). The percentage of symptom-free 24-hour periods, rescue-free 24-hour periods and 24hour period symptom scores was similar for placebo,
GSK2190915 (any dose) and montelukast 10 QD. However, the percentage of symptom-free 24-hour periods and rescue-free 24-hour periods was nominally statistically significantly reduced for FP $100 \mu \mathrm{g}$ compared with placebo. There were nominally statistically significant decreases for GSK2190915 $30 \mathrm{mg}$ QD, montelukast $10 \mathrm{mg}$ QD and FP $100 \mu \mathrm{g}$ versus placebo for 24-hour SABA use. Notable improvements were observed in the placebo group for all efficacy endpoints.

\section{Pharmacokinetics/pharmacodynamics results}

Plasma GSK2190915 levels increased in an approximately dose proportional manner. Geometric mean (inter-subject variability $[\% \mathrm{CV}])$ trough plasma GSK2190915 concentrations were 29 (73) ng/mL following GSK2190915 $10 \mathrm{mg}$ QD, 66 (84) ng/mL following GSK2190915 $30 \mathrm{mg}$ QD, 157 (110) ng/mL following GSK2190915 $100 \mathrm{mg}$ QD, and 370 (111) ng/mL following GSK2190915 $300 \mathrm{mg}$ QD. The sparse plasma GSK2190915 concentration-time data were described by a one-compartment population pharmacokinetic model. Population mean $(\% \mathrm{CV})$ oral clearance was 16 (76) L/hour and apparent volume of distribution was 162 (99) L. Model derived geometric mean (\%CV) area 
Table 1 Demographic and baseline subject characteristics

\begin{tabular}{|c|c|c|c|c|c|c|c|}
\hline & Placebo & $\begin{array}{l}\text { GSK2190915 } \\
10 \mathrm{mg} \text { QD }\end{array}$ & $\begin{array}{c}\text { GSK2190915 } \\
30 \mathrm{mg} \text { QD }\end{array}$ & $\begin{array}{c}\text { GSK2190915 } \\
100 \mathrm{mg} \text { QD }\end{array}$ & $\begin{array}{c}\text { GSK2190915 } \\
300 \mathrm{mg} \text { QD }\end{array}$ & $\begin{array}{c}\text { FP } 100 \mu g \\
\text { BID }\end{array}$ & $\begin{array}{c}\text { Montelukast } \\
10 \mathrm{mg} \text { QD }\end{array}$ \\
\hline & $N=100$ & $N=99$ & $N=100$ & $N=100$ & $N=101$ & $N=103$ & $N=97$ \\
\hline & n (\%) & n (\%) & n (\%) & n (\%) & n (\%) & n (\%) & n (\%) \\
\hline Age in years; Mean [range] & $42.3[12-71]$ & $40.0[12-75]$ & $43.1[12-73]$ & $42.2[12-69]$ & $42.2[12-69]$ & $41.5[12-74]$ & $44.3[15-78]$ \\
\hline BMl in $\mathrm{kg} / \mathrm{m}^{2}$; Mean [range] & $27.5[16-47]$ & $27.5[17-49]$ & $26.8[16-43]$ & $27.8[16-52]$ & 26.9 [19-63] & $27.0[15-55]$ & $27.1[18-54]$ \\
\hline \multicolumn{8}{|l|}{ Sex; n (\%) } \\
\hline Female & $88(88)$ & $91(92)$ & $94(94)$ & $92(92)$ & $93(92)$ & $97(94)$ & $89(92)$ \\
\hline Male & $12(12)$ & $8(8)$ & $6(6)$ & $8(8)$ & $8(8)$ & $6(6)$ & $8(8)$ \\
\hline \multicolumn{8}{|l|}{ Race; n (\%) } \\
\hline White & $82(82)$ & $74(75)$ & $78(78)$ & $78(78)$ & $78(77)$ & $83(81)$ & $76(78)$ \\
\hline Black & $6(6)$ & $12(12)$ & $10(10)$ & $9(9)$ & $9(9)$ & $7(7)$ & $9(9)$ \\
\hline Other & $12(12)$ & $13(13)$ & $12(12)$ & $13(13)$ & $14(14)$ & $13(13)$ & $12(12)$ \\
\hline \multicolumn{8}{|l|}{ Smoking-history; n (\%) } \\
\hline Never smoked & $78(78)$ & $85(86)$ & $83(83)$ & $77(77)$ & $86(85)$ & $86(83)$ & $78(80)$ \\
\hline Current smoker & $12(12)$ & $9(9)$ & $10(10)$ & $11(11)$ & $10(10)$ & $13(13)$ & $13(13)$ \\
\hline Former smoker & $10(10)$ & $5(5)$ & $7(7)$ & $12(12)$ & $5(5)$ & $4(4)$ & $6(6)$ \\
\hline $\mathrm{FEV}_{1}$ in $\mathrm{L}_{\text {; }}$ Mean [range] & $2.0[1.2-3.7]$ & $2.0[1.0-3.3]$ & $1.9[1.1-2.8]$ & $2.0[1.0-3.5]$ & $2.0[1.1-3.4]$ & $2.0[1.0-4.0]$ & $2.0[0.9-3.5]$ \\
\hline$\%$ predicted; Mean [range] & $68.7[51-87]$ & $67.7[50-96]$ & $66.1[50-85]$ & $67.8[51-85]$ & $66.8[51-85]$ & $66.6[37-85]$ & $69.2[50-85]$ \\
\hline Atopy, n\% & $22(22)$ & $30(30)$ & $31(31)$ & $31(31)$ & $35(35)$ & $35(34)$ & $34(35)$ \\
\hline
\end{tabular}

QD Once-daily, FP Fluticasone propionate, BID Twice-daily, BMI Body mass index, $F E V{ }_{1}$ Forced expiratory volume in 1 second.

under the plasma GSK2190915 concentration curve at steady-state was 761 (72) ng.hour/mL for GSK2190915 10 mg QD, 2197 (75) ng.hour/mL for GSK2190915 30 mg QD, 6390 (64) ng.hour/mL for GSK2190915 100 mg QD and 19315 (88) ng.hour/mL for GSK2190915 $300 \mathrm{mg}$ QD.

Exposure to GSK2190915 was associated with a decrease in urinary $\mathrm{LTE}_{4}$ levels (Figure 4). The plasma GSK2190915 concentration versus urinary $\mathrm{LTE}_{4}$ relationship was described by an inhibitory $\mathrm{E}_{\max }$ model. The GSK2190915 plasma concentration associated with 50\% of maximum (100\%) urinary $\mathrm{LTE}_{4}$ inhibition was 33 (113) ng/mL, which equates to a GSK2190915 trough plasma concentration achieved with a dose of $10 \mathrm{mg}$.

\section{Safety and tolerability}

The incidence of adverse events was generally similar between treatment groups (Table 2). The most frequently reported adverse events were headache and nasopharyngitis. Four subjects experienced serious adverse events: dislocated joint ( $n=1$, GSK2190915 $10 \mathrm{mg}$ QD), cranial neuritis ( $\mathrm{n}=1$, GSK2190915 $30 \mathrm{mg}$ QD), small intestine obstruction ( $\mathrm{n}=1$, GSK2190915 $100 \mathrm{mg}$ QD) and injured cartilage ( $\mathrm{n}=1$, FP $100 \mu \mathrm{g}$ BID); none of the events was considered related to study drug by the investigators and all resolved except the cranial neuritis, which led to subject withdrawal from the study. Eleven subjects were discontinued from the study as a consequence of an adverse event. Five of these were considered drug-related: decreased appetite and nausea ( $\mathrm{n}=1$, placebo), dyspnoea ( $\mathrm{n}=1$, GSK2190915 $10 \mathrm{mg}$ QD), extrasystoles and diarrhoea ( $\mathrm{n}=2$, GSK2190915 $300 \mathrm{mg}$ QD) and drug eruption ( $\mathrm{n}=1$, montelukast $10 \mathrm{mg}$ QD). No drug-related adverse events were reported by more than one subject in any treatment group. Fifteen subjects experienced asthma exacerbations across all treatment groups except FP $100 \mu \mathrm{g}$ BID; all episodes were treated with corticosteroids and all exacerbations resolved. One subject became pregnant during the run-in period and was withdrawn before randomisation. No abnormalities in male reproductive health were detected in the follow-up data from the male subjects who participated in the study and underwent follow-up testing. There were no significant or clinically meaningful differences between active treatments and placebo in blood chemistry (including liver function), haematology, urinalysis, vital signs and ECG parameters.

\section{Discussion}

Administration of GSK2190915 for 8 weeks led to numerical increases in mean change from baseline in trough $\mathrm{FEV}_{1}$ at all doses, although none of these achieved statistical significance compared with placebo. A repeated measures sensitivity analysis of the primary endpoint suggested nominal statistical significance for GSK2190915 $30 \mathrm{mg}$ compared with placebo. Improvements were observed in mean day-time symptom scores and mean daytime SABA use for GSK2190915 $30 \mathrm{mg}$ versus placebo. 


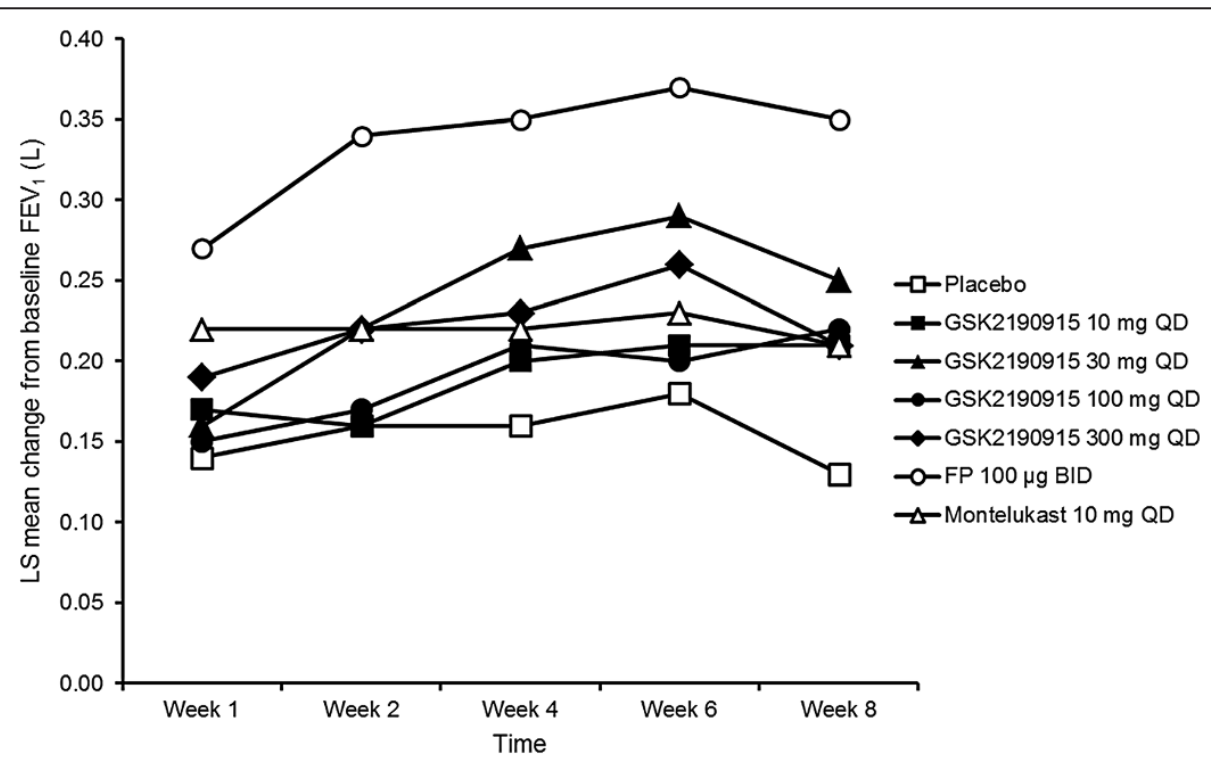

Figure 3 Sensitivity analysis of primary efficacy endpoint. Repeated measures analysis of change from baseline in trough $\mathrm{FEV}_{1}(\mathrm{~L})$. $\mathrm{LS}=$ least squares. 


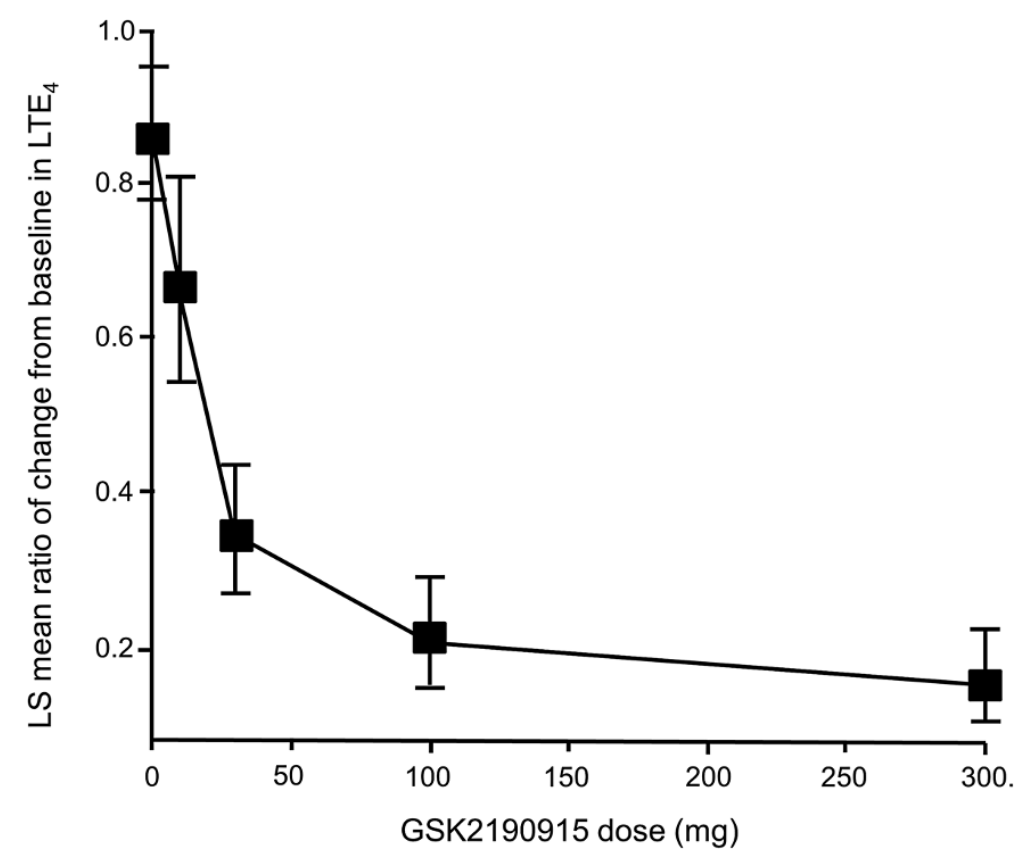

Figure 4 Dose-response plot of urinary $\mathrm{LTE}_{4}$. Ratio of mean change from baseline in urinary $\mathrm{LTE}_{4}$. Error bars represent $95 \%$ confidence interval. LS = least squares.

No additional benefit was gained by administration of GSK2190915 doses greater than $30 \mathrm{mg}$. The results obtained in this study suggest that a GSK2190915 dose of $10 \mathrm{mg}$ QD may be sub-optimal and furthermore suggest that the $\mathrm{FEV}_{1}$ dose-response across the range investigated (30-300 mg) was markedly flat. This was in contrast to the plasma GSK2190915 concentrations that showed increases with dose, and the urinary $\mathrm{LTE}_{4}$ levels that decreased in a dose/GSK2190915 plasma concentrationdependent manner. These pharmacokinetic and pharmacodynamic observations were associated with high inter-subject variability but were comparable to previously reported clinical studies [18].

The results reported in this study population suggest that inhibition of FLAP may not provide further improvement on efficacy endpoints beyond those achieved with cysLT receptor antagonism. However, this requires further evaluation in specifically designed clinical trials. In patients with severe asthma, where neutrophil inflammation plays a significant role in disease pathology, zileuton has demonstrated improvements in lung function. Although zileuton is the only LT synthesis inhibitor currently available, its use has been limited by the need to monitor hepatic enzyme levels and the high frequency of dosing [16]. The findings from the current study indicate that GSK2190915 could play a similar role to zileuton in terms of its anti-LT production activity.

Urinary $\mathrm{LTE}_{4}$ levels decreased in a manner dependent on GSK2190915 dose/GSK2190915 plasma concentration, confirming that GSK2190915 is capable of inhibiting

Table 2 Summary of most frequently reported adverse events ( $>1 \%$ of all subjects who received GSK2190915)

\begin{tabular}{|c|c|c|c|c|c|c|c|c|}
\hline & Placebo & $\begin{array}{c}\text { GSK2190915 } \\
10 \text { mg QD }\end{array}$ & $\begin{array}{c}\text { GSK2190915 } \\
30 \text { mg QD }\end{array}$ & $\begin{array}{c}\text { GSK2190915 } \\
100 \mathrm{mg} \text { QD }\end{array}$ & $\begin{array}{l}\text { GSK2190915 } \\
300 \mathrm{mg} \text { QD }\end{array}$ & $\begin{array}{c}\text { Total } \\
\text { GSK2190915 }\end{array}$ & $\begin{array}{l}\text { FP } 100 \mu g \\
\text { BID }\end{array}$ & $\begin{array}{c}\text { Montelukast } \\
10 \mathrm{mg} \mathrm{QD}\end{array}$ \\
\hline & $N=100$ & $N=99$ & $\mathrm{~N}=100$ & $N=100$ & $N=101$ & $N=400$ & $N=103$ & $\mathrm{~N}=97$ \\
\hline & n (\%) & n (\%) & n (\%) & n (\%) & n (\%) & n (\%) & n (\%) & n (\%) \\
\hline Any event & $20(20)$ & $25(25)$ & $20(20)$ & $20(20)$ & $24(24)$ & $89(22)$ & $25(24)$ & $24(25)$ \\
\hline Headache & $3(3)$ & $7(7)$ & $2(2)$ & $4(4)$ & $4(4)$ & $17(4)$ & $9(9)$ & $9(9)$ \\
\hline Nasopharyngitis & $5(5)$ & $6(6)$ & $3(3)$ & $2(2)$ & $5(5)$ & $16(4)$ & $5(5)$ & $5(5)$ \\
\hline Cough & $1(1)$ & $2(2)$ & $2(2)$ & $2(2)$ & $2(2)$ & $8(2)$ & $3(3)$ & $1(1)$ \\
\hline Nausea & $1(1)$ & $2(2)$ & $1(1)$ & $1(1)$ & $3(3)$ & $7(2)$ & $1(<1)$ & 0 \\
\hline Oropharyngeal pain & $1(1)$ & $1(1)$ & 0 & $2(2)$ & $3(3)$ & $6(2)$ & $1(<1)$ & $1(1)$ \\
\hline Back pain & 0 & $1(1)$ & $2(2)$ & $2(2)$ & $1(<1)$ & $6(2)$ & 0 & $2(2)$ \\
\hline
\end{tabular}

$Q D$ Once-daily, FP Fluticasone propionate, BID Twice-daily. 
the 5-LO pathway in subjects with persistent asthma. GSK2190915 achieved 50\% of maximum estimated inhibition of urinary $\mathrm{LTE}_{4}$ at a plasma concentration of $33 \mathrm{ng} / \mathrm{mL}$, which is comparable to the value estimated from earlier studies in healthy subjects (unpublished observations). However, reduced $\mathrm{LTE}_{4}$ was only associated with small and non-statistically significant changes in $\mathrm{FEV}_{1}$ from baseline. Thus, the measures of efficacy of the present study failed to demonstrate a dose-response for GSK2190915 on $\mathrm{FEV}_{1}$ even though the pharmacokinetic data confirmed that an increase in drug exposure was associated with a dose-dependent decrease in the levels of urinary $\mathrm{LTE}_{4}$.

Previous dose-ranging studies with anti-LT agents have also shown poor relationships between urinary $\mathrm{LTE}_{4}$ concentrations and changes in $\mathrm{FEV}_{1}$ [20-22]. A study of MK-0633, a 5-LO inhibitor, showed that inhibition of urinary $\mathrm{LTE}_{4}$ of approximately $90 \%$ was associated with a relatively modest change in $\mathrm{FEV}_{1}$ from baseline: $0.20 \mathrm{~L}$ compared with $0.13 \mathrm{~L}$ for placebo in change from baseline over the last 4 weeks of a 6 -week treatment period [21]. Zileuton led to significant increases in $\mathrm{FEV}_{1}$ after treatment for 4 weeks (change from baseline: $0.32 \mathrm{~L}$; 95\% CI: $0.16,0.48)$ but the levels of inhibition of urinary $\mathrm{LTE}_{4}$ achieved were only $39 \%$ [22]. Therefore, the results obtained in our study where inhibition of urinary LTE $_{4}$ was associated with a small change from baseline in $\mathrm{FEV}_{1}$ (0.13 L for placebo and 0.18-0.23 L for GSK2190915) are consistent with previous studies and support the hypothesis that changes in $\mathrm{LTE}_{4}$ and $\mathrm{FEV}_{1}$ may not always be highly correlated.

GSK2190915 was well-tolerated and there were no differences between treatment groups in the frequency of adverse events. Previous studies of zileuton and MK0633 suggest that these molecules may have less than optimal safety and tolerability profiles as liver abnormalities were identified $[16,21]$. In one study with MK-0633, a planned extension to the study was terminated prematurely and the patients discontinued because of apparent dose-related increases in alanine aminotransferase and aspartate aminotransferase levels [21]. No such abnormalities were observed in the current study.

As the original protocol allowed recruitment of both male and female subjects, 56 males received at least one dose of study medication. However, interim histopathological assessments of the male reproductive tract from toxicology studies in rats, which were reported during the conduct of the study, revealed testicular toxicity at high exposure levels of GSK2190915. In a proportion of the rats receiving the maximum feasible dose of GSK2190915 (1000 mg/kg/day), testicular atrophy and hypospermia in the epididymides were observed. The reasons behind this finding were unclear at the time and, therefore, it was decided to withdraw all males and only recruit female subjects for the remainder of the study. Although the study population was predominantly female (92\%), the results obtained in the study appear applicable to both genders as, generally, no gender-specific results have been described in asthma treatment.

There is no clear reason that can explain why subjects in the placebo group demonstrated such a high response in the primary and secondary efficacy endpoints. Although there were improvements within active treatment groups, the placebo response may have limited the opportunity for demonstrating significant changes in the efficacy endpoints for active treatments relative to placebo. It is possible that, as the study population was comprised of subjects with relatively mild asthma controlled with a SABA, some subjects improved spontaneously and others are likely to have benefited from the frequent contact with clinical staff and the greater attention received, which may be particularly relevant in a mild asthmatic population.

It could be questioned why no statistically significant difference between GSK2190915 and placebo was achieved for the primary efficacy endpoint, particularly as the outcome measure defined was the most appropriate for a dose range study in asthma. The observed response to FP, the positive control, indicated that the enrolled population was capable of improvement in terms of their lung function response. Furthermore, the study was well designed, incorporated a relatively wide dose range and active comparator and placebo arms were included in the design. It seems to be the case that there are areas in which the design of a randomised trial may result in gaps in the evidence they collect [23]. In particular, randomised trials include tightly controlled and wellcharacterised populations, tend to be limited in the number of outcomes evaluated and are relatively short in duration [23]. For chronic conditions such as asthma, concerns exist regarding the external validity of the data gathered in randomised trials and the ability to extrapolate these data to the heterogeneous patient population treated in everyday clinical practice.

\section{Conclusions}

In subjects with persistent asthma, efficacy was demonstrated for GSK2190915 $30 \mathrm{mg}$ compared with placebo in mean day-time symptom scores and mean day-time SABA use. No additional improvement on efficacy endpoints was gained by administration of GSK2190915 doses greater than $30 \mathrm{mg}$. GSK2190915 was associated with a clear and dose-dependent reduction in urinary $\mathrm{LTE}_{4}$. However, this did not translate into a statistically significant improvement in mean trough $\mathrm{FEV}_{1}$ at Week 8 compared with placebo, although the presence of a notable placebo response may have hindered the assessment of the response to active treatment. The effect of 
GSK2190915 for primary and secondary efficacy endpoints was comparable with montelukast but less than low dose FP. GSK2190915 was generally safe and welltolerated. The GSK2190915 $30 \mathrm{mg}$ dose appears to be the most appropriate for study in future clinical trials.

\section{Additional files}

Additional file 1: Study treatments administered. Table detailing the study treatments administered following the double-dummy design.

Additional file 2: Summary of secondary efficacy parameters.

Summary of the analysis of the secondary efficacy endpoints of the study.

\section{Competing interest}

Richard MA Follows, Shu-Yen Ho, Claire L Ambery, and Barbara A McQuade are GlaxoSmithKline employees. Neil G Snowise and Kevin Smart were employed by GlaxoSmithKline at the time the study was conducted.

\section{Authors' contribution}

RMAF, SYH and CLA participated in the conception and design of the study, and in the analysis and interpretation of the data. NGS and BAM contributed to the conduct of the study, protocol amendment and the analysis and interpretation of the data. KS contributed to analysis and interpretation of the data. All authors have made critical revisions of draft versions of the manuscript and approved the final manuscript.

\section{Acknowledgements}

We thank Marie Duggan for managing the study and Michelle Fry for data management. Justin Cook, PhD, and Severina Moreira, PhD, from Niche Science and Technology (Richmond-upon-Thames, United Kingdom) provided writing and editorial support to the development of this manuscript and were paid for these services by GlaxoSmithKline. This study was funded by GlaxoSmithKline.

\section{Author details}

'GlaxoSmithKline, Stockley Park West, Uxbridge, Middlesex UB11 1BT, UK. ${ }^{2}$ GlaxoSmithKline, 5 Moore Drive, Research Triangle Park, NC 2770, USA. ${ }^{3}$ GlaxoSmithKline, Gunnels Wood Road, Stevenage Herts, SG1 2NY, UK. ${ }^{4}$ Current address: Vectura Group plc, One Prospect West, Chippenham, Wiltshire SN14 6FH, UK. ${ }^{5}$ Current address: Roche Products Limited, 6 Falcon Way, Shire Park, Welwyn Garden City AL7 1TW, UK.

Received: 11 December 2012 Accepted: 15 May 2013

Published: 17 May 2013

\section{References}

1. Funk CD: Prostaglandins and leukotrienes: advances in eicosanoid biology. Science 2001, 294:1871-1875.

2. Miyahara N, Miyahara S, Takeda K, Gelfand EW: Role of the LTB4/BLT1 pathway in allergen-induced airway hyperresponsiveness and inflammation. Allergol Int 2006, 55:91-97.

3. Hicks A, Monkarsh SP, Hoffman AF, Goodnow R Jr: Leukotriene B4 receptor antagonists as therapeutics for inflammatory disease: preclinical and clinical developments. Expert Opin Investig Drugs 2007, 16:1909-1920.

4. Barnes NC, Piper PJ, Costell JF: Comparative effects of inhaled leukotriene C4, leukotriene D4 and histamine in normal subjects. Thorax 1984, 39:500-504.

5. Lewis RA: Leukotrienes and other products of the 5-lipoxygenase pathway. Biochemistry and relation to pathobiology in human diseases. N Engl J Med 1990, 323:645-655.

6. Wenzel SE: The role of leukotrienes in asthma. Prostaglandins Leukot Essent Fatty Acids 2003, 69:145-155.

7. Peters-Golden M, Henderson WR: Leukotrienes. N Engl J Med 2007, 357:1841-1854

8. Dworski R, Fitzgerald GA, Oates JA, Sheller JR: Effect of oral prednisone on airway inflammatory mediators in atopic asthma. Am J Respir Crit Care Med 1994, 149:953-959.
9. Wenzel SE, Szefler SJ, Leung DY, Sloan SI, Rex MD, Martin RJ: Bronchoscopic evaluation of severe asthma. Persistent inflammation associated with high dose glucocorticoids. Am J Respir Crit Care Med 1997, 156:737-743.

10. Vachier I, Bonnans C, Chavis C, Farce M, Godard P, Bousquet J, Chanez P: Severe asthma is associated with a loss of $L X 4$, an endogenous antiinflammatory compound. J Allergy Clin Immunol 2005, 115:55-60.

11. Afonso PV, Janka-Junttila M, Lee YJ, McCann CP, Oliver CM, Aamer KA, Losert W, Cicerone MT, Parent CA: LTB4 is a signal-relay molecule during neutrophil chemotaxis. Dev Cell 2012, 22:1079-1091.

12. Vachier I, Kumlin M, Dahlen SE, Bousquet J, Godard P, Chanez P: High levels of urinary leukotriene $\mathrm{E} 4$ excretion in steroid treated patients with severe asthma. Respir Med 2003, 97:1225-1229.

13. Jatakanon A, Uasuf C, Maziak W, Lim S, Chung KF, Barnes PJ: Neutrophilic inflammation in severe persistent asthma. Am J Respir Crit Care Med 1999, 160:1532-1539.

14. From the Global Strategy for Asthma Management and Prevention, Global Initiative for Asthma (GINA); 2011. http://www.ginasthma.org/.

15. Amlani S, Nadarajah T, Mclvor RA: Montelukast for the treatment of asthma in the adult population. Expert Opin Pharmacother 2011, 12:2119-2128

16. Berger W, De Chandt MT, Cairns CB: Zileuton: clinical implications of 5-Lipoxygenase inhibition in severe airway disease. Int I Clin Pract 2007, 61:663-676

17. Lorrain DS, Bain G, Correa LD, Chapman C, Broadhead AR, Santini AM, Prodanovich PP, Darlington JV, Stock NS, Zunic J, King CD, Lee C, Baccei CS, Stearns B, Roppe J, Hutchinson JH, Prasit P, Evans JF: Pharmacology of AM803, a novel selective five-lipoxygenase-activating protein (FLAP) inhibitor in rodent models of acute inflammation. Eur J Pharmacol 2010, 640:211-218.

18. Bain G, King C, Schaab K, Rewolinsk M, Norris V, Ambery C, Bentley J, Yamada M, Santini AM, van de Wetering de Rooij J, Stock N, Zunic J, Huitchinson JH, Evans JH: Pharmacodynamics, pharmacokinetics, and safety of GSK2190915, a potent, novel 5-lipoxygenase-activating protein inhibitor. Br J Clin Pharmacol 2013, 75:779-790.

19. National Institutes of Health $(\mathrm{NIH})$ : Guidelines for the Diagnosis and Management of Asthma - Expert Panel Report 3. NIH Publication No. 07-4051. Bethesda, MD: U.S. Department of Health and Human Services; 2007. http://www.nhlbi.nih.gov/guidelines/asthma/.

20. García-Marcos L, Schuster A, Pérez-Yarza EG: Benefit-risk assessment of antileukotrienes in the management of asthma. Drug Saf 2003, 26:483-518

21. Wasfi YS, Villarán C, de Tilleghem Cle B, Smugar SS, Hanley WD, Reiss TF, Knorr BA: The efficacy and tolerability of MK-0633, a 5-lipoxygenase inhibitor, in chronic asthma. Respir Med 2012, 106:34-46.

22. Israel E, Rubin P, Kemp JP, Grossman J, Pierson W, Siegel SC, Tinkelman D, Murray JJ, Busse W, Segal AT, Fish J, Kaiser HB, Ledford D, Wenzel S, Rosenthal R, Cohn J, Lanni C, Pearlman H, Karahalios P, Drazen JM: The effect of inhibition of 5-lipoxygenase by zileuton in mild-to-moderate asthma. Ann Intern Med 1993, 119:1059-1066.

23. Price D, Chisholm A, van der Molen T, Roche N, Hillyer EV, Bousquet J: Reassessing the evidence hierarchy in asthma: evaluating comparative effectiveness. Curr Allergy Asthma Rep 2011, 11:526-538.

doi:10.1186/1465-9921-14-54

Cite this article as: Follows et al:: Efficacy, safety and tolerability of GSK2190915, a 5-lipoxygenase activating protein inhibitor, in adults and adolescents with persistent asthma: a randomised dose-ranging study. Respiratory Research 2013 14:54. 